
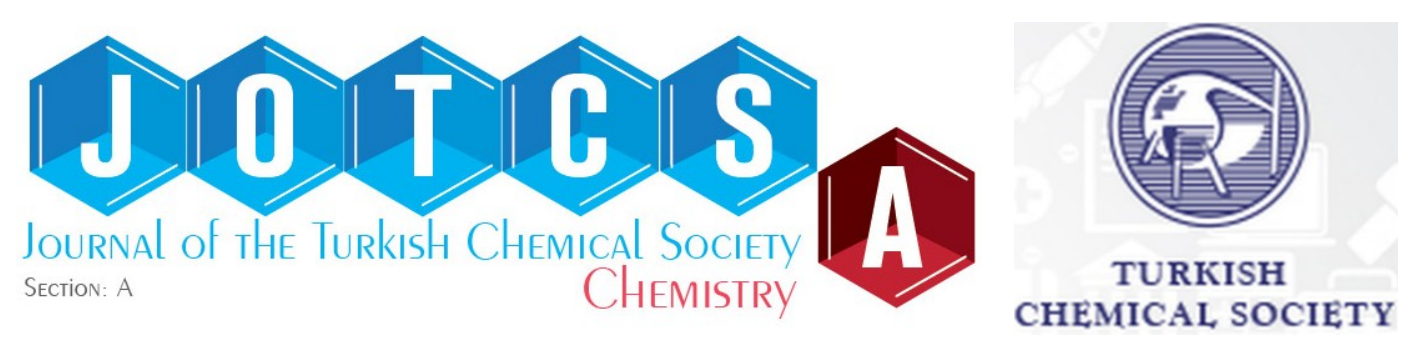

\title{
A comprehensive study on electrospinning of poly (vinyl alcohol): effects of the TCD, applied voltage, flow rate, and solution concentration
}

\author{
Gokhan Acik* \\ Piri Reis University, Faculty of Science and Letters, Department of Chemistry, Tuzla, TR-34940 Istanbul, \\ Turkey
}

Abstract: In the present work, the electrospun fibers of poly (vinyl alcohol) (PVA) are fabricated from its solution in water under ambient conditions, and the effect of altered working parameters used such as tipto-collector distance (TCD), applied voltage, flow rate and solution concentration on the average diameter and frequency distribution of them is deeply discussed. The mean electrospun PVA fiber diameters and its distributions are estimated by a combination of the Scanning electron microscope (SEM) and the ImageJ analyzer program, which is extensively utilized in respective sciences. The achieved results from the experiments indicate that higher both TCD and applied voltage used decrease the average electrospun PVA fiber diameter. Conversely, when the flow rate or solution concentration is increased, diameters of achieved fibers are soared. It is anticipated that this comprehensive study will be beneficial to academia and industry working on potential PVA fiber applications.

Keywords: electrospinning; poly (vinyl alcohol); scanning electron microscope; working parameters.

Submitted: May 22, 2020. Accepted: June 11, 2020.

Cite this: Acik G. A comprehensive study on electrospinning of poly (vinyl alcohol): effects of the TCD, applied voltage, flow rate, and solution concentration. JOTCSA. 2020;7(2):609-16.

DOI: https://doi.org/10.18596/jotcsa.741452.

*Corresponding author. E-mail: (gacik@pirireis.edu.tr), Tel: (+90 21658100 50/1665), Fax: (+90 216 581 0051).

\section{INTRODUCTION}

To date, with the increasing global interest, considerable research efforts have been generated aiming at the production of fibers of both natural or synthetic polymers and inorganic materials for a remarkable range of application areas such as chemistry, medicine, material science, nanotechnology, textile, and so on (1-8). In this respect, the most well-known fiber production methods mentioned in the literature are mechanical drawing, self-assembly, hydrothermal processing, electrospinning, melt blowing, phase separation, template synthesis, extraction, vapor-phase polymerization, and solvent casting $(7,9)$. Among them, especially, electrospinning process, which can be applied as the spinning of the wet or dry solution, melt at elevated temperatures, gel, and emulsion has emerged as an efficient, affordable, and most extensively utilized method. A typical relatively simple electrospinning instrument setup consists of a metallic nozzle, syringe pump, voltage supply, and collector (screen or roller) apparatus for making fibers with diameters ranging from few nanometers to a few hundred nanometers. Generally, the setup is designed for feeding ranging from $\mu \mathrm{L} \cdot \mathrm{h}^{-1}$ to $\mathrm{mL} \cdot \mathrm{min}^{-1}$ and making $0-40 \mathrm{kV}$ voltage value over distances of $0-40 \mathrm{~cm}$ between the nozzle and collector. During the experiments, after the voltage is imposed, the polymer droplet that emerged on the nozzle is transformed into a conical shape (known as Taylor cone) via potential difference formed. The continuously elongated polymeric jet that is ejected from the pendant droplet by overcoming the surface tension of the spinning solution reaches the collector while the solvent is evaporated. Many types of electrospinning parameters such as TCD distance, applied voltage, flow rate and needle diameter (process parameters), the solvent used, concentration, viscosity and conductivity of the solution, molecular weight, solubility, glass-transition temperature, 
chain entanglement density of polymer (molecular parameters), relative humidity, pressure and temperature of surrounding (ambient parameters) can strongly influence the various properties and structure of the final product (10). Producing the long lengths fibers, providing the higher surface area to volume ratio and superior mechanical properties, and allowing the functionalization and tunable surface morphologies are some of the advantages that it provides. Moreover, the electrospinning process offers many advantages due to its ease controllability, economic competitiveness, and versatility in broad range potential fields such as biosensor technology, enzyme immobilization, optoelectronics, filtration, tissue engineering, drug delivery, wound dressings, self-cleaning, environmental remediation, and etc (11).

PVA is biodegradable and biocompatible, semicrystalline and water-soluble industrial polymer, and is available as entirely (91-99\%), moderately (92-96\%) or partially (87-89\%) hydrolyzed forms produced by sequential polymerization of vinyl acetate monomer and hydrolysis of achieved poly (vinyl acetate) (PVAC). Depending on its hydrolysis degree, the mechanical, chemical, and physical properties of PVA, such as rigidity, tensile strength, flexibility, solubility, crystallinity, and biodegradability are in a wide range $(9,12)$. As an FDA-approved material, PVA based products have been in use for advanced widespread industrial, commercial, food, and medical applications, including textile, paper, antimicrobial packaging, tissue engineering, wound dressing, contact lenses, and drug delivery on account of its excellent features (13-15). Hence, PVA has piqued the interest of industry and academia for many years due to the information mentioned above. Furthermore, PVA aqueous solution can be easily prepared and electrospun; thus, the present comprehensive study will be promising for the scientists working on different PVA fiber applications.

This study aims to demonstrate the influence of a variety of electrospinning parameters on the average diameter and frequency distribution of electrospun PVA fibers to create a detailed database for scientists and industry workers. The morphological analysis of PVA fibers was studied through scanning electron microscopy and accurate image processing.

\section{EXPERIMENTAL SECTION}

\section{Materials}

During the experiments, the utilized commercially available fully hydrolyzed Poly (vinyl alcohol) (PVA, $M_{\mathrm{w}}=60,000 \mathrm{~g} \cdot \mathrm{mol}^{-1}$ ) was purchased from Merck (Darmstadt, Germany). To prepare the PVA electrospinning solutions, water $\left(\mathrm{H}_{2} \mathrm{O}\right.$, ultrapure grade) procured from Merck (Steinheim, Germany) was used. Rectangular microscope cover glasses used as fiber accumulated substrate $(3 \times 1$ inch) were supplied from ISOLAB (Istanbul, Turkey). No necessary purification and distillation procedures were implemented for all chemicals and solvents used.

\section{Preparation of electrospinning solutions}

Commercially available, fully hydrolyzed PVA was dissolved in distilled $\mathrm{H}_{2} \mathrm{O}$ by vigorously stirring on a magnetic stirrer at $60{ }^{\circ} \mathrm{C}$ for $3 \mathrm{~h}$ to obtain 11,13 , and $15 \%(w / w)$ solutions to be used in the electrospinning process for determining the effect of solution concentration.

\section{Electrospinning procedure of PVA}

To fabricate the electrospun fibers of PVA achieved previously from its solutions at different concentrations, a transparent chamber of electrospinning setup equipped with a syringe pump (NE-500, New Era Pump Systems Inc., Turkey), a high voltage power source (Electrosis, PW1010, Turkey). A $5 \mathrm{~mL}$ plastic syringe with metal needle and rectangular microscope cover glasses, which are cleaned two times with chromic acid solutiondistilled water cycle, having $3 \times 1$ inch dimensions stuck aluminum plate collector was utilized. To conduct the detailed investigation on the effect of different parameters on electrospinning, 15, 20 and $25 \mathrm{kV}$ are applied on PVA solutions at 11,13 and $15 \%$ concentrations when they are fed by $0.6,1.0$ and $1.4 \mathrm{~mL} \cdot \mathrm{h}^{-1}$ rates fixed to 7,10 and $13 \mathrm{~cm}$ TCDs, separately. During the experiments, relative humidity and temperature were $41 \%$ and $24.1{ }^{\circ} \mathrm{C}$ and almost constant. After conducting the electrospinning experiments, electrospun fiber coated glasses were kept in a desiccator to remove the residual $\mathrm{H}_{2} \mathrm{O}$ for $72 \mathrm{~h}$ at room temperature to be used for SEM analysis. Electrospinning time was 5 min for all experiments.

\section{Characterizations}

Surface morphologies and characteristics of each PVA fibers obtained by using different electrospinning parameters were examined with the help of scanning electron microscopy (SEM, at 10.0 kV, JEOL JSM-6335F, Tokyo, Japan, after coating with Pt (Platin) by sputter coater device (Polaron SC7620, East Sussex, United Kingdom). The average diameters and histograms of fibers were determined to utilize Image $\mathrm{J}$ processing software application (National Institutes of Health; USA).

\section{RESULTS AND DISCUSSION}

The electrospinning variables influencing the full range of features of the fibers have motivated the investigators in many types of research for use in diverse applications (16, 17). In our study, electrospinning parameters mentioned elsewhere in the literature were changed; a variety of optimum spinning conditions were utilized to achieve the 
smooth and uniform PVA fibers without the free beads. Some SEM images selected were presented in Figures (a)-(e) to provide seeing the micrometer scale, unimodal, cylindrical, and beadless structure and smooth surface of the achieved PVA fibers to readers. As can be easily seen from the $\times 2,500$ and 10,000 zoomed SEM images, while the flow rate and solution concentration fixed, if the applied voltage or TCD is increased to higher values, it is visibly evident that average diameter of the obtained fibers decreases to lower, as expected. Moreover, the obtained PVA fiber structures were almost the same in these conditions.

The primary purpose of this study was to provide researchers with a comprehensive overview of how several electrospinning parameters would affect the average fiber diameters of PVA fibers and their distribution. In this respect, the detailed Table 1 and some selected histograms (Figures 2(a)-(e) were presented.

The effect of polymer solution concentration on the diameter of electrospun fibers has been studied in the literature by several groups. Indeed, the uniaxial stretching of a charged jet, which can strongly be affected by the solution concentration mentioned in the Introduction part, is the essential phenomenon in the electrospinning technique. Based on the literature results, it is well-known that the increasing solution concentration caused to an increase in solution viscosity and formation of uniform fibers after the critical chain entanglement value, and increasing average diameter of fibers as well $(18,19)$. Firstly the effect of solution concentration on the average diameter of electrospun PVA fibers was investigated, and PVA solution concentration was varied from 11 to $15 \%$ $(w / w)$. After the solution concentration was increased from 11 to 13 or $15 \%$, the mean diameter of PVA fibers gradually was increased. The obtained results were in good agreement with the literature, as tabulated in Table 1 (20).

As stated in previous information, feeding or flow rate is another parameter that affected the resulted average diameter of fibers in the electrospinning process. Higher flow rate produces fibers with relatively larger diameter or vice versa due to the formation of a higher accumulated amount of suspended droplet on the spinneret that can lead to insufficient time to elongation of polymer chains (21). In our study, the flow rate was varied in the range of 0.6 and $1.4 \mathrm{~mL} . \mathrm{h}^{-1}$. One can see in Table 1 , after the used flow rate was increased, the mean diameter of electrospun PVA fibers was increased, supporting the above-stated results achieved from concentration changes.

The reverse influences solution concentration and flow rate effects were obtained by altering both TCD and applied voltage parameters. The prevention of bead formation and adjusting the fiber diameter can be taken control by selecting the optimal TCD providing adequate time to complete solvent evaporation from the fibers before reaching the collector (19). As can be seen in Figures 1(a)-(e) and 2(a)-(e), and Table 1 as well, while the electrospinning TCD changed from 7 to $13 \mathrm{~cm}$, the mean PVA fiber diameter decreased probably due to the reason mentioned above. On the other hand, the applied voltage that is known as driving force to jet initiation during electrospinning was the other examined parameter for solution electrospinning of PVA. In this respect, in our present study, PVA solution having different either concentrations and feed rates or varied TCD was electrospun with 15 , 20 , or $25 \mathrm{kV}$ applied voltage. The average diameter of all the electrospun fibers achieved as uniform without bead formation decreased gradually with the increasing applied voltage from 15 to $25 \mathrm{kV}$, supporting the trend of mean diameter change when the TCD increased from 7 to $13 \mathrm{~cm}$. These applied voltage changing results combined with the TCD alteration results were attributable to producing ultrafine or nanofibers resulting from higher electrostatic force to stretch the jet during electrospinning. Also, the observed results were in good agreement with the literature, as expected (22). 

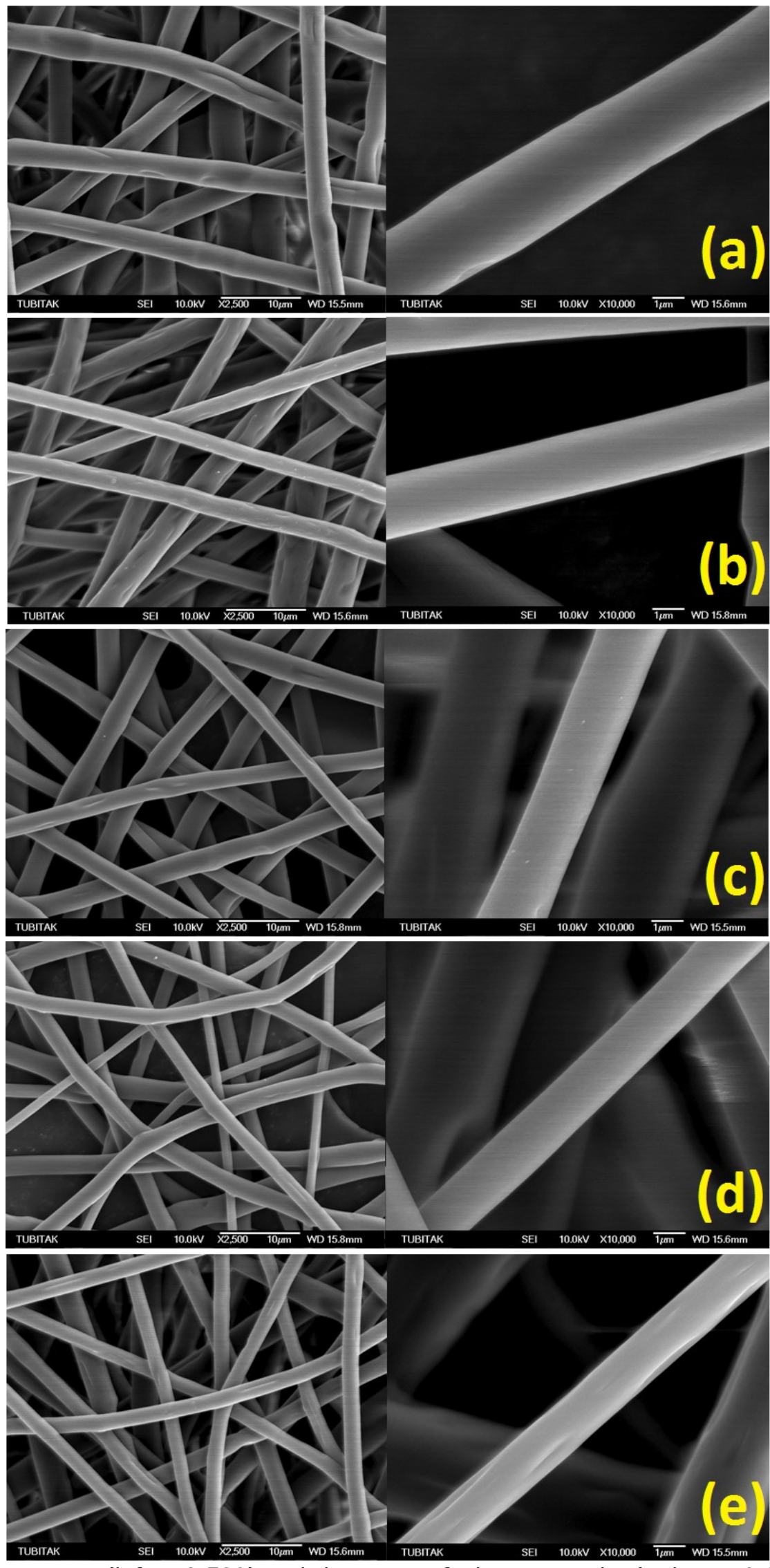

Figure 1. The SEM images (left, $\times 2,500$ ) and their magnified micrographs (right, $\times 10,000$ ) of electrospun PVA fibers obtained from $1 \mathrm{~mL} \cdot \mathrm{h}^{-1}$ flow rate, $13 \%$ (w/w) solution concentration, (a) $15 \mathrm{kV}$ (7 cm TCD) (b) $20 \mathrm{kV}$ (7 cm TCD) (c) $25 \mathrm{kV}$ (7 cm TCD) (d) $25 \mathrm{kV}$ (10 cm TCD) (e) $25 \mathrm{kV}$ (13 cm TCD) applied voltages. 

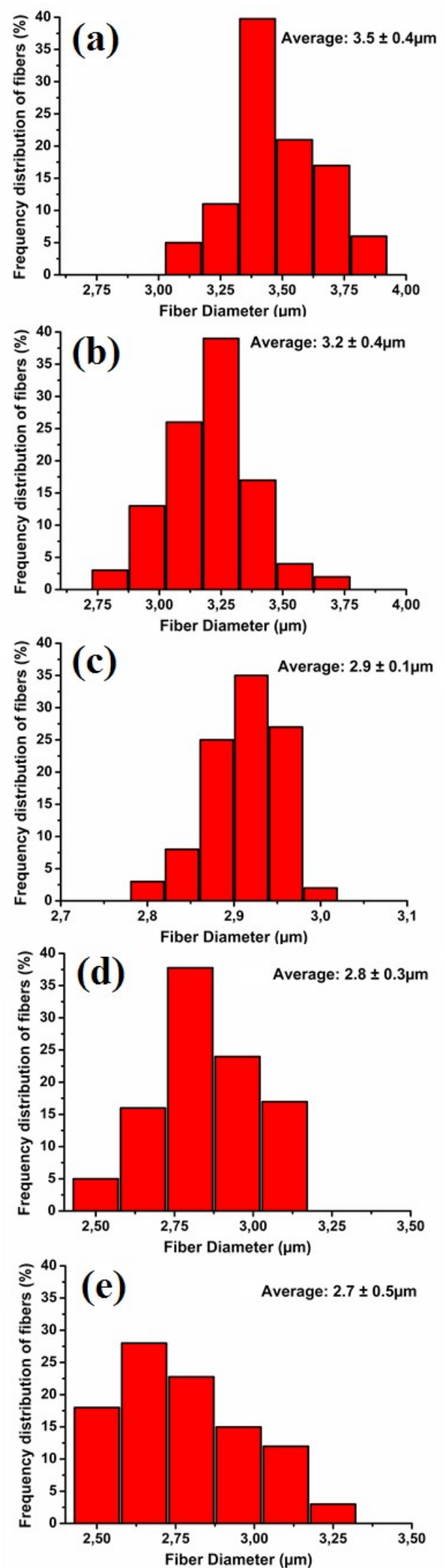

Figure 2. Frequency distribution and average diameter histograms of electrospun PVA fibers obtained from $1 \mathrm{~mL} \cdot \mathrm{h}^{-1}$ flow rate, $13 \%$ (w/w) solution concentration, (a) $15 \mathrm{kV}$ (7 cm TCD) (b) $20 \mathrm{kV}$ (7 cm TCD) (c) 25 $\mathrm{kV}$ (7 cm TCD) (d) $25 \mathrm{kV}$ (10 cm TCD) (e) $25 \mathrm{kV}$ (13 cm TCD) applied voltages. 
Table 1. The data of experiments carried out at each variable of tip-to-collector distance, solution concentration, applied voltage, and solution flow rate.

\begin{tabular}{|c|c|c|c|c|c|c|}
\hline \multirow[b]{2}{*}{$\begin{array}{l}\text { Experiment } \\
\text { No }\end{array}$} & \multicolumn{3}{|c|}{$\begin{array}{c}\text { Tip-to-Collector Distance, } x=7,10 \text { and } \\
13 \\
(\mathrm{~cm})\end{array}$} & \multicolumn{3}{|c|}{$\begin{array}{l}\text { Average Fiber Diameter } \pm s t d \text { dev. } \\
(\mu \mathrm{m})\end{array}$} \\
\hline & $\begin{array}{l}\text { Solution Conc. } \\
(\% w / w)\end{array}$ & $\begin{array}{l}\text { Applied } \\
\text { Voltage } \\
(\mathbf{k V})\end{array}$ & $\begin{array}{l}\text { Solution } \\
\text { Flow } \\
\text { Rate } \\
(\mathbf{m L . h})\end{array}$ & $x=7 \mathrm{~cm}$ & $x=10 \mathrm{~cm}$ & $x=13 \mathrm{~cm}$ \\
\hline 1 & 11 & 15 & 0.6 & $3.1 \pm 0.3$ & $3.0 \pm 0.1$ & $2.9 \pm 0.7$ \\
\hline 2 & 11 & 15 & 1.0 & $3.1 \pm 0.5$ & $3.0 \pm 0.5$ & $3.0 \pm 0.1$ \\
\hline 3 & 11 & 15 & 1.4 & $3.1 \pm 0.9$ & $3.1 \pm 0.4$ & $3.0 \pm 0.9$ \\
\hline 4 & 11 & 20 & 0.6 & $2.9 \pm 0.4$ & $2.7 \pm 0.1$ & $2.6 \pm 0.8$ \\
\hline 5 & 11 & 20 & 1.0 & $2.9 \pm 0.9$ & $2.9 \pm 0.2$ & $2.8 \pm 0.8$ \\
\hline 6 & 11 & 20 & 1.4 & $3.0 \pm 1.0$ & $3.0 \pm 0.6$ & $2.9 \pm 0.9$ \\
\hline 7 & 11 & 25 & 0.6 & $2.7 \pm 0.5$ & $2.6 \pm 0.9$ & $2.4 \pm 0.2$ \\
\hline 8 & 11 & 25 & 1.0 & $2.8 \pm 0.1$ & $2.7 \pm 0.7$ & $2.6 \pm 0.7$ \\
\hline 9 & 11 & 25 & 1.4 & $2.8 \pm 0.5$ & $2.9 \pm 0.5$ & $2.9 \pm 0.1$ \\
\hline 10 & 13 & 15 & 0.6 & $3.4 \pm 0.3$ & $3.2 \pm 0.3$ & $3.1 \pm 0.1$ \\
\hline 11 & 13 & 15 & 1.0 & $3.5 \pm 0.4$ & $3.4 \pm 0.6$ & $3.2 \pm 0.4$ \\
\hline 12 & 13 & 15 & 1.4 & $3.6 \pm 0.3$ & $3.4 \pm 0.2$ & $3.3 \pm 0.3$ \\
\hline 13 & 13 & 20 & 0.6 & $3.0 \pm 0.4$ & $2.9 \pm 0.3$ & $2.8 \pm 0.3$ \\
\hline 14 & 13 & 20 & 1.0 & $3.2 \pm 0.4$ & $3.1 \pm 0.5$ & $2.9 \pm 0.4$ \\
\hline 15 & 13 & 20 & 1.4 & $3.3 \pm 0.4$ & $3.2 \pm 0.3$ & $3.1 \pm 0.6$ \\
\hline 16 & 13 & 25 & 0.6 & $2.8 \pm 0.2$ & $2.7 \pm 0.3$ & $2.5 \pm 0.3$ \\
\hline 17 & 13 & 25 & 1.0 & $2.9 \pm 0.1$ & $2.8 \pm 0.3$ & $2.7 \pm 0.5$ \\
\hline 18 & 13 & 25 & 1.4 & $3.0 \pm 0.5$ & $2.9 \pm 0.1$ & $2.8 \pm 0.7$ \\
\hline 19 & 15 & 15 & 0.6 & $3.6 \pm 0.5$ & $3.5 \pm 0.4$ & $3.3 \pm 0.5$ \\
\hline 20 & 15 & 15 & 1.0 & $3.7 \pm 0.4$ & $3.6 \pm 0.3$ & $3.5 \pm 0.3$ \\
\hline 21 & 15 & 15 & 1.4 & $3.8 \pm 0.3$ & $3.6 \pm 0.7$ & $3.6 \pm 0.1$ \\
\hline 22 & 15 & 20 & 0.6 & $3.4 \pm 0.5$ & $3.2 \pm 0.5$ & $3.1 \pm 0.3$ \\
\hline 23 & 15 & 20 & 1.0 & $3.4 \pm 0.8$ & $3.3 \pm 0.7$ & $3.2 \pm 0.1$ \\
\hline 24 & 15 & 20 & 1.4 & $3.6 \pm 0.4$ & $3.6 \pm 0.8$ & $3.5 \pm 0.9$ \\
\hline 25 & 15 & 25 & 0.6 & $3.1 \pm 0.3$ & $2.9 \pm 0.3$ & $2.8 \pm 0.1$ \\
\hline 26 & 15 & 25 & 1.0 & $3.2 \pm 0.5$ & $3.1 \pm 0.7$ & $3.1 \pm 0.2$ \\
\hline 27 & 15 & 25 & 1.4 & $3.2 \pm 0.8$ & $3.2 \pm 0.2$ & $3.1 \pm 0.9$ \\
\hline
\end{tabular}

\section{CONCLUSION}

In this study, the influence of electrospinning parameters such as polymer solution concentration, solution flow rate, tip-to-collector distance, and applied voltage was investigated deeply for the electrospun PVA fiber morphology, frequency distribution, and average diameter by conducting 27 separate experiments. Fiber properties such as fiber mean diameter, morphology, frequency distribution, etc. can be fully controlled with an appropriate combination of process, solution, and environmental parameters in the electrospinning process for the desired functions. The effect of these different variables on the resulted PVA fibers was tried to be explained logically by correlating with the previously published electrospinning literature papers. Depending on the attained results from the present comprehensive study, PVA fibers thus offer many attributions to scientists working on PVA fiber applications.

\section{FUNDING}

This research did not receive any specific grant from funding agencies in the public, commercial, or notfor-profit sectors.

\section{CONFLICT OF INTEREST}

The authors declare that they have no known competing financial interests or personal relationships that could have appeared to influence the work reported in this paper.

\section{REFERENCES}

1. Sankar S, Sharma CS, Rath SN, Ramakrishna S. Electrospun fibers for recruitment and differentiation of stem cells in regenerative medicine. Biotechnology journal. 2017;12(12):1700263. 
2. Lee C-G, Javed $H$, Zhang D, Kim J-H, Westerhoff $\mathrm{P}$, $\mathrm{Li} \mathrm{Q}$, et al. Porous electrospun fibers embedding $\mathrm{TiO} 2$ for adsorption and photocatalytic degradation of water pollutants. Environmental science \& technology. 2018;52(7):4285-93.

3. Dzenis $Y$. Spinning continuous fibers for nanotechnology. Science. 2004;304(5679):1917-9.

4. Toshniwal L, Fan Q, Ugbolue SC. Dyeable polypropylene fibers via nanotechnology. Journal of applied polymer science. 2007;106(1):706-11.

5. Sanjay M, Arpitha G, Naik LL, Gopalakrishna K, Yogesha B. Applications of natural fibers and its composites: An overview. Natural Resources. $2016 ; 7(3): 108-14$.

6. Neves A, Bointon TH, Melo L, Russo S, De Schrijver I, Craciun MF, et al. Transparent conductive graphene textile fibers. Scientific reports. 2015;5:9866.

7. Acik G, Kamaci M, Cansoy CE. Superhydrophobic EVA copolymer fibers: the impact of chemical composition on wettability and photophysical properties. Colloid and Polymer Science. 2018;296(11):1759-66.

8. AÇIK G, Kamaci M, ÖZATA B, ÖZEN CANSOY CE. Effect of polyvinyl alcohol/chitosan blend ratios on morphological, optical, and thermal properties of electrospun nanofibers. Turkish Journal of Chemistry. 2019;43(1).

9. Teixeira MA, Amorim MTP, Felgueiras HP. Poly (Vinyl Alcohol)-Based Nanofibrous Electrospun Scaffolds for Tissue Engineering Applications. Polymers. 2020;12(1):7.

10. Cui W, Li X, Zhou S, Weng J. Investigation on process parameters of electrospinning system through orthogonal experimental design. Journal of applied polymer science. 2007;103(5):3105-12.
11. Acik G, Altinkok C. Polypropylene microfibers via solution electrospinning under ambient conditions. Journal of Applied Polymer Science. 2019;136(45):48199.

12. Chahal S, Kumar A, Hussian FSJ. Development of biomimetic electrospun polymeric biomaterials for bone tissue engineering. A review. Journal of Biomaterials Science, Polymer Edition. 2019;30(14):1308-55.

13. Arslan M, Acik G, Tasdelen MA. The emerging applications of click chemistry reactions in the modification of industrial polymers. Polymer Chemistry. $2019 ; 10(28): 3806-21$.

14. Yu Z, Li B, Chu J, Zhang P. Silica in situ enhanced PVA/chitosan biodegradable films for food packages. Carbohydrate polymers. 2018;184:214-20.

15. Liu B, Xu H, Zhao H, Liu W, Zhao L, Li Y. Preparation and characterization of intelligent starch/PVA films for simultaneous colorimetric indication and antimicrobial activity for food packaging applications. Carbohydrate polymers. 2017;157:842-9.

16. Lin J, Ding B, Yang J, Yu J, Sun G. Subtle regulation of the micro-and nanostructures of electrospun polystyrene fibers and their application in oil absorption. Nanoscale. 2012;4(1):176-82.

17. Huang L, Bui NN, Manickam SS, McCutcheon JR. Controlling electrospun nanofiber morphology and mechanical properties using humidity. Journal of polymer science part B: Polymer physics. 2011;49(24):1734-44.

18. Oliveira JE, Mattoso LH, Orts WJ, Medeiros ES. Structural and morphological characterization of micro and nanofibers produced by electrospinning and solution blow spinning: a comparative study. Advances in Materials Science and Engineering. 2013;2013.

19. Shao $H$, Fang J, Wang $H$, Lin $T$. Effect of electrospinning parameters and polymer concentrations on mechanical-to-electrical energy conversion of randomlyoriented electrospun poly (vinylidene fluoride) nanofiber mats. RSC advances. 2015;5(19):14345-50. 
20. Motamedi AS, Mirzadeh $H$, Hajiesmaeilbaigi $F$, Bagheri-Khoulenjani $S$, Shokrgozar M. Effect of electrospinning parameters on morphological properties of PVDF nanofibrous scaffolds. Progress in biomaterials. 2017;6(3):113-23.

21. Zong X, Kim K, Fang D, Ran S, Hsiao BS, Chu B. Structure and process relationship of electrospun bioabsorbable nanofiber membranes. Polymer. 2002;43(16):4403-12.
22. Liu Y, Dong L, Fan J, Wang R, Yu JY. Effect of applied voltage on diameter and morphology of ultrafine fibers in bubble electrospinning. Journal of Applied Polymer Science. $2011 ; 120(1): 592-8$. 